\title{
A Model of Large-Scale Evolution of Complex Food Webs
}

\author{
C. Guill* \\ Institute of Condensed Matter Physics, Darmstadt University of Technology \\ Hochschulstraße 6, D-64289 Darmstadt, Germany
}

\begin{abstract}
A simple model of biological evolution of community food webs is introduced. This model is based on the niche model, which is known to generate model food webs that are very similar to empirical food webs. The networks evolve by speciation and extinction. Co-extinctions due to the loss of all prey species are found to play a major role in determining the longterm shape of the food webs. The central aim is to design the model such that the characteristic parameters of the niche model food webs remain in realistic intervals. When the mutation rule is chosen accordingly, it is found that food webs with a complex, biologically meaningful structure emerge and that the statistics of extinction events agrees well with that observed in the paleontological data.
\end{abstract}

Key words: stochastic model, food web structure, complexity, extinction record AMS subject classification: 92D40, 92D15, 65C99

\section{Introduction}

Food webs are the networks of feeding relationships in an ecosystem. It is still an open question what determines the characteristic patterns in the structure of food webs in habitats as different as marine, freshwater, or terrestrial systems [27]. In several recent publications the evolutionary history of ecological communities has been proposed to play a major role in determining regularities in the topology of the food webs. In these works, models are developed that either assign feeding links by referring to phylogenetic constraints and thus implicitly include the evolutionary history of food webs [7] or that construct food webs in a dynamical process [37, 38]. These models focus

*E-mail: guill@fkp.tu-darmstadt.de 
on the reproduction of statistical topological quantities of empirical food webs, and the emergent topological characteristics of the networks are explained by the assumptions made in the evolutionary algorithm. However, statistical data concerning the evolutionary history of the food webs are less extensively studied (some information on the strong fluctuations and intermittent extinction events observed in the evolutionary time series are for example provided in [39]).

The process of community evolution has received more attention from another direction. It has been found that the fossil record displays intermittent, self-similar extinction patterns [16, 18, 43] that suggest that biological evolution might be a self-organized critical phenomenon. Indeed, it has been found that the statistics of extinction events are compatible with a power law distribution $[32,33,28,41]$, which is a further indication for self-organized critical behavior. The exponent of the distribution was estimated to $\alpha=2.0 \pm 0.2[28,33]$.

A variety of models have been developed [24, 3, 42, 2, 40], in part accompanied by analytical calculations $[31,11]$, to address this topic. They mainly analyze the statistics of co-evolutionary avalanches that are found to follow power law distributions. In [42, 2, 40], extinction avalanches are recorded, which allows direct comparison of the models' predictions with the paleontological data, and the exponents of the distributions generally are in good agreement. In some of the cited studies species lifetime distributions or power spectra are analyzed, too, and always found to be in line with the theoretical framework of self-organized criticality. However, it should be noted that the observed power law distributions can be explained equally well in a model that explicitly does not invoke self-organized criticality [29]. For reviews on models studying evolution in the context of SOC, see [30, 12].

In most of the models mentioned above, the species are arranged in a network that defines the interactions between them. Exceptions are the model of Bak and Sneppen [3] where only nearest neighbor interactions on a one-dimensional, periodic lattice are considered and Newman's model of mass extinction [29] where interactions are completely neglected. Although it is sometimes claimed that evolution of ecosystems is investigated, the structure of these networks is not paid much attention to. In fact, only in one case the structure of the network is ecologically motivated [2].

The issue of the present work is to overcome this shortcoming and to develop a model of largescale evolution of food webs that have a biologically realistic topology not only at initialization but during the whole evolutionary process. To this end, a "basic model" is introduced that is based on the niche model [49]. The basic model is then modified in two comprehensible steps. By this it is shown that an arbitrary choice of extinction and mutation rules can lead to unrealistic network structures and avalanche distributions. The importance to adjust the rules of the evolutionary process in such a way that constraints from the underlying model are accounted for is stressed.

The niche model is chosen as underlying topological network model because it is known to be capable of producing model food webs that are very similar to empirically food webs. It has the further advantage of being very simple. This permits a good understanding of the evolutionary process and to systematically test assumptions that enhance the stability and complexity of the evolving networks. Complexity is mainly measured by the length of food chains and by the relative occupancy of trophic levels. In this work, the trophic level of a species is defined as its shortest distance to the external resources (the energetic basis of the food web) via a chain of feeding links. 
In the following sections, the static niche model is briefly introduced and the basic evolutionary algorithm acting on it is presented. It is shown that it leads to very unstable networks that regularly collapse and that often have a trivial structure. When the evolutionary algorithm is refined to include more meaningful conditions regarding the extinction probabilities, the networks become more stable, but food chains remain short. In both cases it is shown that the record of extinction avalanches deviates from the empirical pattern. By further adjusting the speciation procedure, more complex networks emerge and the extinction statistics are in good agreement with empirical data.

\section{The Topological Model}

The rules by which trophic links between species are assigned are taken from the niche model [49]. This topological model uses only two parameters as input, food web size $S$ and directed connectance $C=\frac{L}{S^{2}}$ ( $L$ is the number of links in the network) to produce artificial food webs that agree well with empirical food webs in a large range of structural quantities like number of top predators, standard deviation of vulnerability distribution, or degree of omnivory [50].

Each species is assigned a niche value $n$ that is drawn with constant probability distribution from the interval $[0,1]$. Species $i$ is a predator of all species $j$ whose niche values $n_{j}$ fall in a range with width $n_{i} r_{i}, 0<r_{i} \leq 1$ and center $c_{i}$ (see fig. 1). The center $c_{i}$ of the feeding range $c_{i} \pm \frac{n_{i} r_{i}}{2}$ is drawn uniformly from the interval $\left[\frac{n_{i} r_{i}}{2}, n_{i}\right]$. In this work, the parameter $r_{i}$ is called the relative width of the feeding range. It is drawn from a $\beta$-distribution with expected value $E_{r}=2 C$, therefore the expected value of the absolute width of the feeding range is $E_{n r}=C$, in accordance with the desired value of connectance. This requires the parameter $\beta$ of the probability distribution to depend on $C$ according to $\beta=\frac{1-2 C}{2 C}$, while the second parameter of the distribution, usually labeled $\alpha$, is set to 1 . Since up to half the feeding range may be greater than the niche value of the predator, the model permits looping and cannibalism. The central assumption of the niche model is that the species in a habitat can be ordered on a one-dimensional niche axis in such a way that all prey species of a given predator fall in closed ranges on this axis. The one-dimensional niche space has to be understood as a simplifying approximation to the observation that empirical food webs are close to perfect intervality [45]. An explanation for this observation is proposed by another model [7].

As in most of the stochastic models of large-scale evolution of ecosystems [3, 42, 2, 40], species are treated as binary objects, either present or absent from the web. They do not have any additional intrinsic property like a population size.

\section{Basic evolutionary algorithm}

The evolution of the model food webs is described as a stochastic process with discrete time steps. One time step is defined by the introduction of a new species as a variation of an existing one (speciation), or, when there is no suitable parent species present, as an invading species with random attributes. Also, in each time step there is a certain probability that one species in the network 


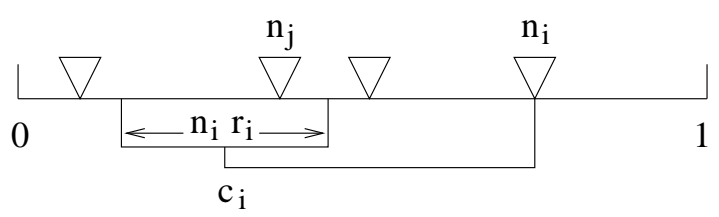

Figure 1: Diagram of the niche model (after [49]). Inverted triangles represent species; species $j$ is a prey of species $i$.

becomes extinct. To make sure that the network does not increase indefinitely, this extinction probability becomes 1 when a maximum number of species $\left(S_{\max }\right)$ is reached. The extinction of species can be understood as the effect of competition among the species in the food web. The resources of an ecosystem are limited and there is only a finite number of ecological niches, each of which is inhabited by only one species (competitive exclusion principle, [20]).

In the static model [49], a species is considered to be a basal species or autotroph when its feeding range is empty. In this study, however, the external resources the autotrophs feed on (like sunlight, water, or chemical nutrients) are explicitly present as a virtual species on the niche axis. Those species that have the external resources in their feeding range are considered as basal species. This deviation from the original model is a necessary refinement for the evolutionary process. Each species has to have an energetic basis, otherwise it will become extinct in the next time step. This basis can be provided either by a direct link to the resources or by a chain of feeding links that ultimately ends in the resources. When the last prey species of a predator becomes extinct the predator also should become extinct instead of suddenly becoming an autotroph.

In detail, the steps of the algorithm are as follows:

1. A niche network is initialized with $S_{\max }$ trophic species and one virtual species that represents the external resources. The parameters $n, r$, and $c$ of the species and thereby the links between them are determined as in the original, static niche model (see Sec. 2.). The external resources are assigned a niche value $n_{R}$ that is slightly larger than $\frac{1}{S_{\max }}$. This value is kept constant in all simulations with the same $S_{\max }$ and it is used as lower bound for the niche values of the trophic species. The width of the feeding range of the resources is set to zero.

2. The trophic level of the species is calculated and species without food source are removed from the network. The external resources are on level 0 . The trophic level of a species is defined as its shortest distance from the resources, measured over chains of feeding links. It is not sufficient to simply remove those species that have an empty feeding range. Since in the niche model, cannibalism and feeding loops are possible, one also has to identify and eliminate species in isolated loops (one or more species preying exclusively on themselves).

3. Random extinction of one species. With probability $P=\frac{S}{S_{\max }}$, one randomly chosen species becomes extinct. Here, $S$ is the number of species that are connected to the network after step 2 of the algorithm. In the basic algorithm, each species has the same chance to die out. The individual extinction probability therefore is $p_{e x t}=\frac{1}{S_{\max }}$. It does not depend on the number of prey or predators the species have. 
4. Introduction of a new species by speciation. Of the remaining species, one is uniformly randomly chosen to give birth to a daughter species. The daughter species $d$ is introduced as a variation of the mother species $m$ with the following parameters:

$$
\begin{aligned}
n_{d} & =n_{m}+\delta_{n} \\
r_{d} & =r_{m}+\delta_{r} \\
c_{d} & =c_{m}+\delta_{c} .
\end{aligned}
$$

$\delta_{n, r, c}$ are three independent random numbers that are drawn with uniform probability from the interval $[-0.05,0.05]$. If one of the daughter species' parameters violates the constraints of the niche model (e.g., $n_{d}>1$ ), the whole set of parameters is rejected and a new one is created. Predator- and prey species of the new species are then determined following the rules of the niche model. The mother species is not removed from the food web and can be chosen again to give birth to another daughter species.

When in the second step all species have been removed, a new species is introduced with completely random parameters. This can be interpreted as an invasion event.

The external resources are neither removed from the network by chance nor can they be chosen as mother species. The algorithm is iterated by returning to step 2. Typical simulation runs are iterated for $10^{6}$ time steps, statistical data for the frequency distribution of extinction avalanches were taken from simulation runs with $10^{8}$ time steps. Simulations become very time consuming and the statistical data obtained are of poor quality when $S_{\max } \gg 10^{2}$. Therefore only simulations with $S_{\max }=50,100$, and 200 have been performed.

In the following it is shown that this simple algorithm leads to either trivial or unstable networks and to unrealistic distributions of the parameters $n, r$, and $c$. Therefore it will be adjusted in two steps to make these distributions more realistic, i.e., to make them follow the constraints of the niche model more closely.

\section{Results obtained with the basic algorithm}

The appearance and extinction of species is governed by stochastic processes in this algorithm, but there are nevertheless several mechanisms that lead to a directed evolution of the networks. First, newly introduced species are not independent of those already present (this would be the case in an invasion model where the parameters $n_{t}, r_{t}$ and $c_{t}$ were completely random). Therefore, after some time all species will have a common ancestor.

A stronger, albeit indirect impact on a correlated evolution of the networks is given by the procedures that lead to the extinction of species. The random extinction (step 3 of the algorithm) affects all species with the same probability, irrespective of their parameter values. It therefore does not influence the development of the network. However, the extinction of species without resources (step 2 of the algorithm) affects only species on the second or any higher trophic level and mainly those that have few prey species due to a small feeding range. 
A typical time series with $S_{\max }=50$ species is shown in figure 2, left panel. Over long periods, the network evolution is characterized by low activity. The number of species fluctuates around a mean value that does not change during time. A closer look at the network reveals that during these periods it has a trivial structure: all species have a direct link to the external resources and there are no links between the species. Therefore, in each time step only one randomly selected species may die out but no secondary extinctions occur.

Although in each time step a new species is introduced, the total number of species does not increase to $S_{\max }$ because there is a finite probability that the new species has an empty feeding range and becomes extinct immediately after appearance. The mean number of species during these periods of low evolutionary activity depends on the niche value of the external resources. The higher $n_{R}$ is, the higher is the probability that the feeding range of the newly introduced species contains the resources and the more species co-exist in the food web on average.

The quasi-static periods are interrupted by sudden "bursts" of evolutionary activity. The species number increases and the network structure becomes more complex as species on higher trophic levels emerge (figure 2, right panel). During the bursts the number of basal species fluctuates but eventually drops to zero which causes the whole food web to collapse. The food web then starts to rebuild from one species with random parameters $n, r$, and $c$. These parameters do not depend on the history of the evolutionary process but they determine to some extend the future development of the network. When the niche value is low or when the feeding range of the new species covers a wide range of the niche axis between the external resources and the species itself, it is likely that the network evolves again into a complex state. If, however, the niche value of the invading species is high and its feeding range covers only a small range on the niche axis around the resources, it is impossible that a direct descendant has any species in its feeding range but the resources. Therefore only species with a direct link to the resources accumulate and the food web once again enters a period of low activity and trivial network structure.

The pattern of long periods with little change in the network composition and sudden bursts of activity is known as punctuated equilibrium [16, 3, 18], which is often accompanied by power laws in the statistics of extinction events. In figure 3, the frequency distribution $N(s)$ of extinction avalanches of size $s$ is analyzed for food webs of various maximal sizes $S_{\max }$. For small avalanches, the distributions indeed resemble power laws $N(s) \propto s^{-\alpha}$. The exponent $\alpha$ is determined by a fit of a Zipf distribution to the cumulative distribution of the avalanche sizes, which gives robust results [48]. It is found to vary slightly between $\alpha=1.6 \pm 0.1\left(S_{\max }=50\right)$ and $\alpha=1.7 \pm 0.1\left(S_{\max }=200\right)$, which is smaller than the empirical value $\alpha=2.0 \pm 0.2$.

As mentioned before, after the complete collapse of a community the system can directly build up a complex food web with multiple trophic levels again. The ultimate fate of the new network, however, again is the complete collapse. Due to this mechanism, the size distributions of extinction events strongly increase at large avalanche sizes. Both the small values of the exponent $\alpha$ and the overrepresentation of extinction avalanches up to the maximal size of the respective food web are not compatible with paleobiological data [32]. 

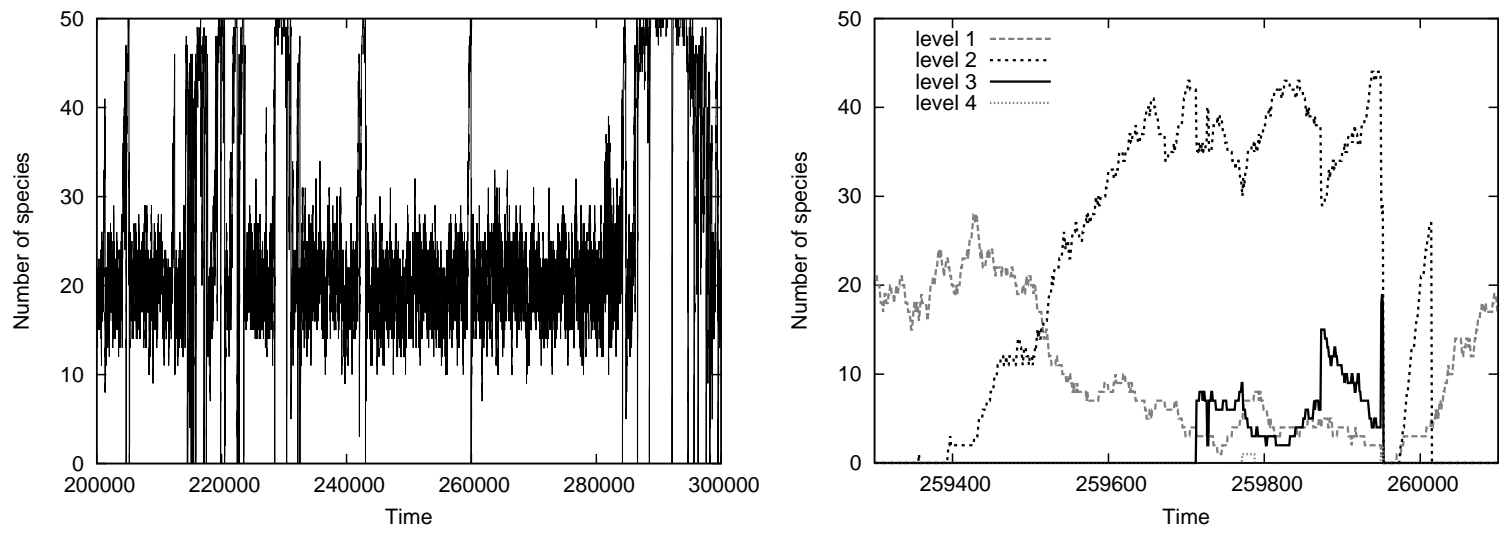

Figure 2: Left panel: Time series of the number of species in a food web with $S_{\max }=50$ species. Long periods of low evolutionary activity, small changes in network size and a trivial network structure are interrupted by "burst" where the species number grows fast but that ultimately lead to the collapse of the whole community. Right panel: Number of species on the different trophic levels during a burst. First, all species are on the first level, then the number of species without direct link to the resources suddenly increases. When the number of species on the first level decreases by chance to 1 , the network is at risk of a total collapse.

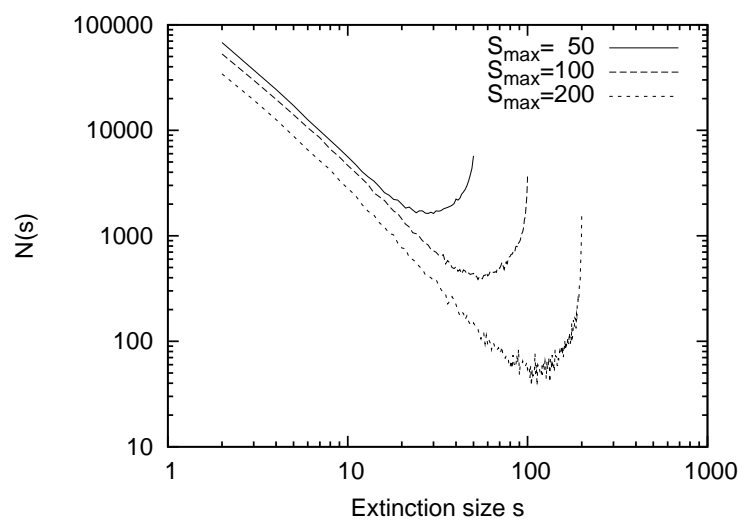

Figure 3: Size distribution of extinction avalanches in networks with $S_{\max }=50,100$, and 200 species. Data have been accumulated from time series with $10^{8}$ time steps. 


\section{Competition for resources}

Given today's prevalence of complex ecological systems, neither the long periods of neutral evolution in trivial network states nor the frequent total or near to total collapses of the food webs once they evolve towards a more complex state resemble a biologically meaningful evolution of ecological communities.

The extinction of all species on one trophic level inevitably leads to the loss of all species on the higher levels. When the depopulation particularly of the first level can be avoided, a more stable evolution of complex food webs (i.e., those with more than one trophic level) can be expected. For this, step 3 of the basic algorithm has to be revised.

Choosing the species that dies out at random with a constant probability implies that each species is in competition with every other species. However, the species of an ecosystem have very different functions and requirements (think of plants, herbivores, and carnivores). While plant species might compete for space on the ground, herbivores might compete for safe nesting sites that provide refuge against carnivores. These additional resources are not explicitly accounted for in the one-dimensional niche model. One can therefore argue that competition acts only between species of one trophic level. This decreases the extinction probability when only few species coexist on a trophic level. In the algorithm, the individual extinction probability is adjusted so that it increases linearly with the number of species on the same trophic level, $S_{j}$, where $j$ is the index of the trophic level ${ }^{\dagger}$. Species on the third and every higher trophic level are pooled in this process because there are only few of them and because they all belong to the group of carnivores.

With this adjustment of the algorithm, the time series of the number of species becomes more regular (figure 4, left panel). In the interval shown, the species number drops less than 10 times below fifty percent of the maximal network size. By this, the evolution of single food webs can be analyzed over long periods of time without the network loosing all memory of its evolutionary history abruptly in a complete collapse.

The distribution of species on the trophic levels (figure 4, right panel) is a practical indicator by which the complexity of the networks can be assessed. For long instances of time, the networks consist of no more than two trophic levels, corresponding to plants and herbivores. Carnivores occur only sporadically in the time series, though in this case even fourth level species can be found. Species on trophic levels higher than the second either become extinct very fast or they change to level two when a plant species originates in their feeding range.

This result can be explained by the distribution of the parameters $n, r$, and $c$ in their accessible space (figure 5, left and center column). The average distributions of the parameters of 2500 niche networks with $S_{\max }=50$ species at initialization and after $2.5 \cdot 10^{4}$ speciation events (which is long enough for the evolving network to loose the memory of its initial state) are compared. While at initialization, the distribution of the niche values $n$ is approximately constant, those of the relative

\footnotetext{
${ }^{\dagger}$ The probability for a species on trophic level $j$ to become extinct in one time step is thereby now given by $p_{\text {ext }, j}=\frac{S}{S_{\max }} \cdot \frac{S_{j}^{2}}{\sum_{j} S_{j}^{2}} \cdot \frac{1}{S_{j}}$. The first factor is the probability that in the corresponding time step one species becomes extinct, while the second factor denotes the probability that a species from trophic level $j$ is chosen. It is quadratic in $S_{j}$ to model density dependent competition. The third factor is the probability for each of the $S_{j}$ species on trophic level $j$ to become extinct, which is the same for all species on the respective level.
} 

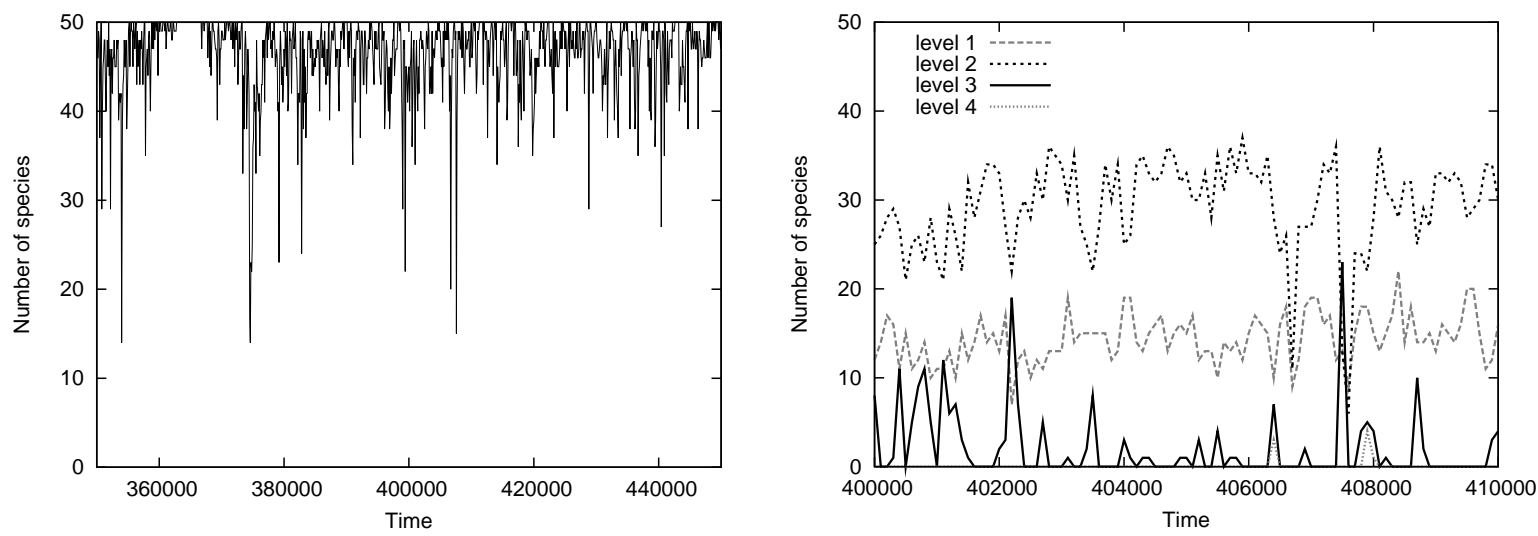

Figure 4: Left panel: Time series of the number of species in a food web with $S_{\max }=50$ species when competition acts between species on the same trophic level. Right panel: Number of species on the different trophic levels. For greater clarity, a shorter interval of the time series is shown and only one in hundred data points is plotted. The number of species on the first level does not decrease to zero, but the network mainly consists of only two trophic levels.

feeding range, $r$, and the feeding center, $c$, are monotonically and nearly linearly decreasing. The small deviations from these general rules at the lower end of the distributions originate from details of the initialization procedure. For example, species that are not connected to the network (mainly those with a very small and thereby empty feeding range) are neglected in the distributions.

In the second column the average distributions of the parameters after $2.5 \cdot 10^{4}$ speciation events are shown. The constant distribution of the niche values and the monotonically decreasing distribution of the relative feeding widths have changed to increasing distributions. The distribution of the feeding centers has a pronounced peak between 0.4 and 0.5 . This means that for sufficiently long times, the evolving food webs are dominated by species whose feeding ranges span nearly the complete niche axis.

This can be explained with the effect of the extinction mechanism mentioned at the beginning of section 4. Species are evolutionary successful, i.e., they have many surviving descendants, if they minimize their extinction probability (which increases the probability to be chosen as mother species several times) and if the daughter species also have a high chance to survive for long times. The first condition is fulfilled for species on the first trophic level (because their resources never become extinct) and for species with a large feeding range. A high fraction of daughter species survives when a small variation of the parameters of the mother species still makes sure that one of the aforementioned conditions is fulfilled. Only if the niche value of the mother species is close to 1 and only if its feeding range spans the complete niche axis a species maximizes its own survivability and that of its descendants in a manner that is independent of the niche values of the other species (that in this argument are only considered as potential prey). It is obvious that this restrains the emergence of species on trophic levels higher than the second since wide feeding ranges increase the probability to have at least one prey species on the first level in it. 

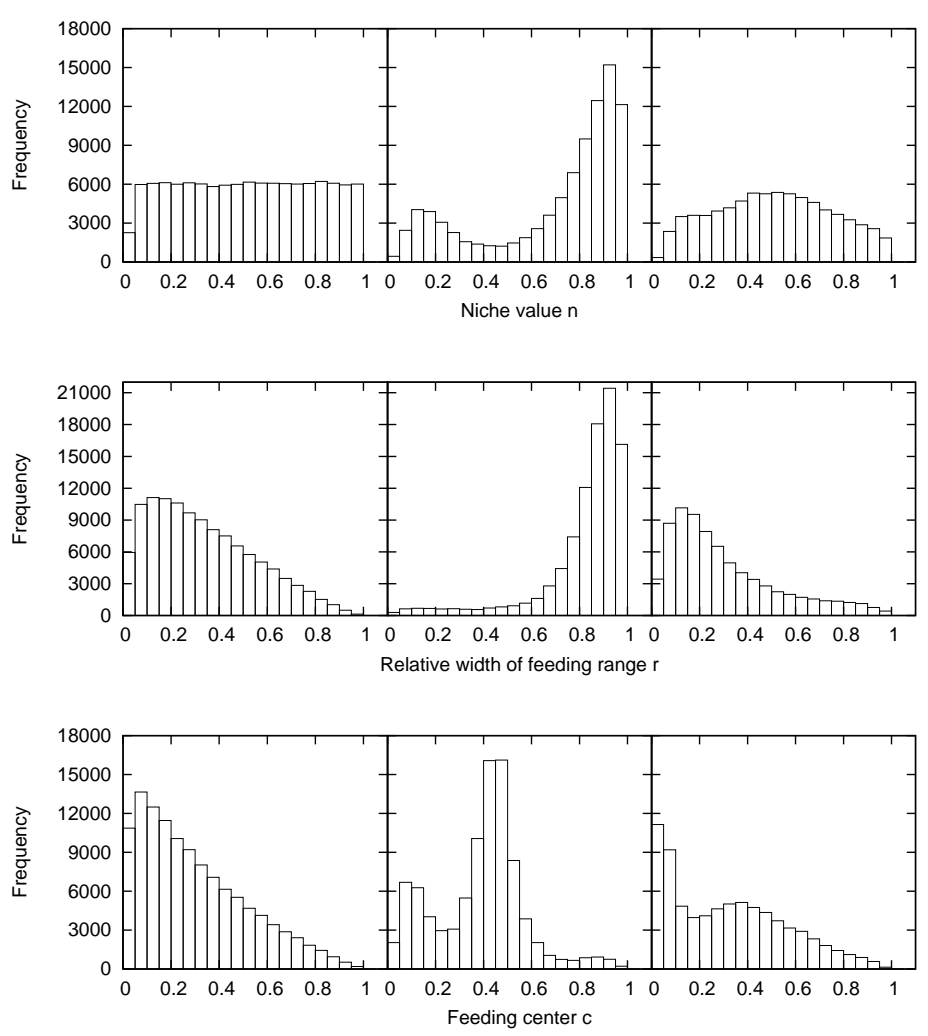

Figure 5: Distributions of the parameters $n, r$, and $c$ in 2500 niche networks with $S_{\max }=50$ species. Left column: initial distributions, center column: after $2.5 \cdot 10^{4}$ time steps with symmetric speciations, right column: after $2.5 \cdot 10^{4}$ time steps with asymmetric speciations.

The frequency distributions $N(s)$ of extinction avalanches with size $s$ are now cut off at avalanche sizes much smaller than $S_{\max }$ (figure 6). The peak at large avalanche sizes either disappears completely $\left(S_{\max }=50\right)$ or becomes much smaller $\left(S_{\max }=100\right.$ or 200$)$. While this indicates a more stable and biologically more reasonable network evolution, the distributions follow power laws only over about one order of magnitude on the abscissa. This makes the approximation of the exponents $\alpha$ less reliable and consequently they vary more between the three data sets. The best fits are $\alpha=1.6 \pm 0.1$ for $S_{\max }=50, \alpha=1.8 \pm 0.1$ for $S_{\max }=100$, and $\alpha=2.2 \pm 0.2$ for $S_{\max }=200$. Only in the last case the exponent is in the range of those found in other models $[42,2]$ and in the paleobiological data [32].

\section{Asymmetric speciation}

Although the speciation procedure (step 4 of the algorithm) is symmetric by construction, i.e., the probability of the daughter species' parameters to be smaller than those of the mother species is the same than being larger, the evolutionary process favors on average higher parameter values, 


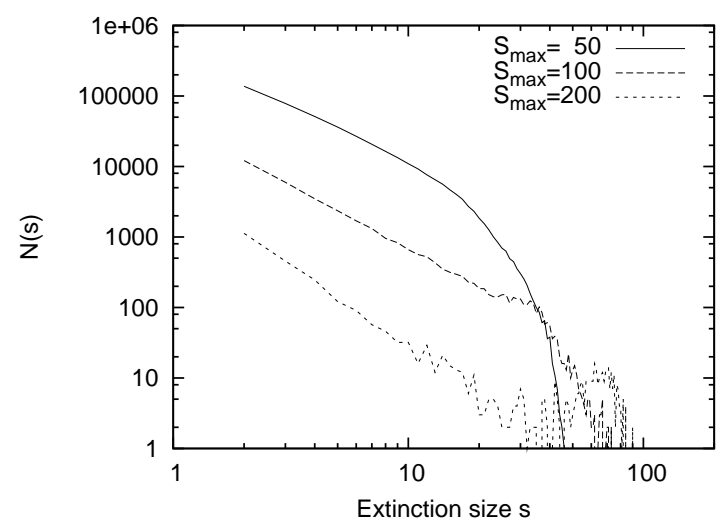

Figure 6: Size distribution of extinction avalanches in networks with $S_{\max }=50,100$, and 200 species when competition acts between species on the same trophic level. Data have been accumulated from time series with $10^{8}$ time steps.

as discussed. This leads to food webs with low structural complexity. A neutral evolution of the parameters over long periods of time that does not change the shape of the parameter distributions much should, on the other hand, preserve the relative occupancy of the trophic levels at initialization. A simple method to achieve this is to introduce a drift on the stochastic variations of the daughter species' parameters towards lower values:

$$
\begin{aligned}
n_{d} & =n_{m}+\delta_{n}-\zeta_{n} \\
r_{d} & =r_{m}+\delta_{r}-\zeta_{r} \\
c_{d} & =c_{m}+\delta_{c}-\zeta_{c} .
\end{aligned}
$$

$\zeta_{n, r, c}$ are three constants that can be used independently for fine tuning of the model. This modification of the algorithm is also biologically plausible: It represents degeneration which in the absence of evolutionary pressure would slowly shift the characteristics of the species into one direction. With $S_{\max }=50, \zeta_{n}=0.005, \zeta_{r}=0.006$ and $\zeta_{c}=0$ the parameter distributions in the right column of figure 5 are obtained. The distribution of the niche values still is not constant, but it is nearly symmetrical around 0.5 . The distributions of the other two parameters now also qualitatively mirror the trends of the initial distribution.

The asymmetric speciation process has a positive effect on the complexity of the evolving network structure. In figure 7 the time series of the total species number (left panel) and the number of species on the trophic levels (right panel) is given. Species on the third trophic level persist for several thousand time steps and their number is comparable to those on the second level. However, the food web still sometimes evolves through phases of trivial network structure, but these are now much shorter than those obtained with the basic algorithm.

To analyze the topology of the evolving food webs in more detail, the distributions of generality, $g(k)$, and vulnerability, $v(k)$, with $k$ the number of prey or predator species, respectively, are investigated. In figure 8, the degree distributions are plotted for $S_{\max }=50,100$, and 200 

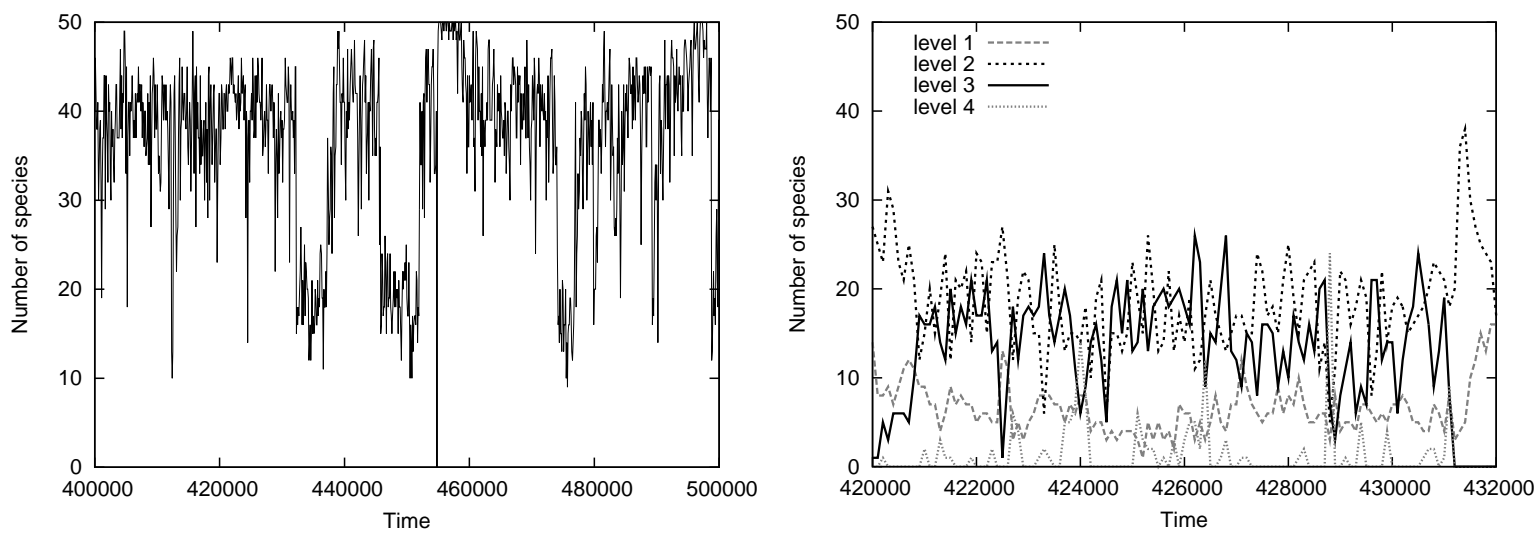

Figure 7: Competition acts only between species on the same trophic level and speciation is asymmetric. Left panel: Time series of the number of species in a food web with $S_{\max }=50$ species. Right panel: number of species on the different trophic levels. Again only one in hundred data points is plotted. Species on trophic levels higher than the second persist in the food web for several thousand time steps.

and compared with the corresponding distributions obtained from the original niche model and from the algorithm with symmetric speciation rule. The generality distribution in the static niche model decays approximately exponentially, which explains the success of the model in reproducing empirical food web patterns [44]. The vulnerability distribution however has a characteristic bimodal shape, which does not reproduce empirical patterns perfectly (in analytic studies that analyze the limit of large species numbers and low connectance, this is not seen $[5,6])$. The peak at the upper end of the vulnerability distributions becomes more pronounced the larger $S$ is. This over-representation of species with a high number of predators explains the low stability of niche model food webs under deterministic population dynamics [23].

When the speciation rule is symmetric, both generality- and vulnerability distributions are nearly flat, indicating once again that in this case, the generated networks have little in common with realistic food webs. However, when speciation is asymmetric, both degree distributions are strongly skewed towards low values and decay fast. Remarkably, the vulnerability distributions obtained from the evolving food webs seems to fit empirical patterns even better than the original, static niche model [23].

The resemblance of empirical food web patterns is further supported by a number of statistical quantities evaluated for networks with $S_{\max }=50,100$, and 200, see table 1 . The average values of connectance, number of links per species, fractions of top, intermediate, and basal species, as well as the fraction of cannibalistic species are well in the range of values observed in a set of large marine food webs [15] and trends like increasing number of links per species with increasing total species number are correctly reproduced. But it also has to be noted that some topological quantities are not reproduced that well. For example, the mean trophic level is lower in the present 

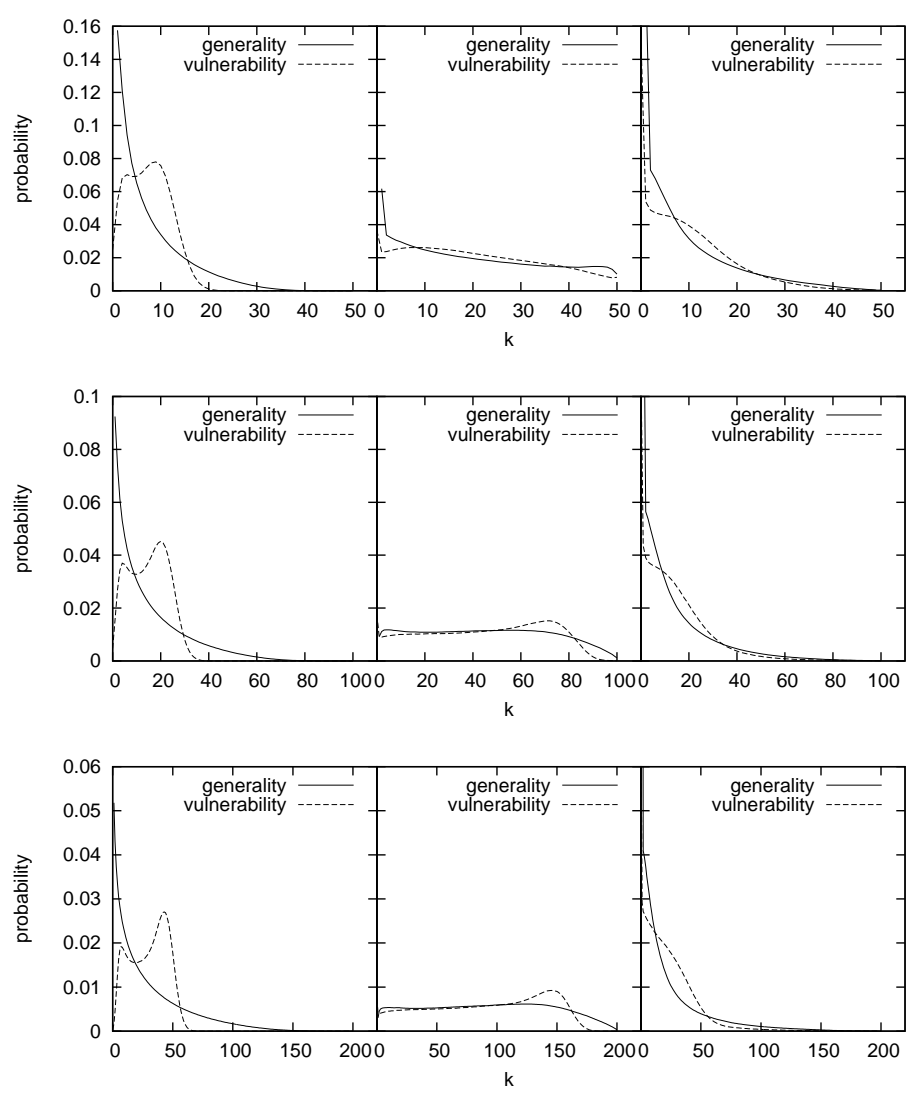

Figure 8: Generality- and vulnerability distributions in networks with $S_{\max }=50$ (top), 100 (middle), and 200 (bottom) species. Left column: degree distributions in the initial networks, center column: distributions in evolving networks with symmetric speciations, right column: distributions in evolving networks with asymmetric speciations.

study than in the empirical webs. This is likely to be because of different ways of computing trophic levels. Here, the shortest distance to the external resources has been evaluated, but often the average over different supply routes is taken. Also, the model over-estimates the fraction of omnivorous species: The values found here are at the upper end of the values reported in [15]. Finally, the fraction of basal species has to be revisited critically again. In empirical studies, the bottom layer of food webs is often not well resolved or many species are lumped together to form so-called trophic species. The present study therefore very likely underestimates the fraction of basal species in ecological communities, despite the seemingly good agreement with existing empirical data.

As an illustrative example of the complexity of the food webs emerging from the algorithm as described so far, the graph of a network is given in figure 9. The species are ordered according to their trophic level, but they do not feed only on species on the next lower level as in a simple layer model (which was the network topology chosen in [2]). Omnivory (feeding of prey on several 
trophic levels) occurs on all levels but the first and is more common among carnivores. The thin horizontal lines between species on one trophic level depict intraguild predation links (which can be seen as a special case of omnivory), but their start- and endpoints are not well resolved in this representation of the graph. Between the third and the fourth trophic level, the network contains several loops and mutual feeding links.

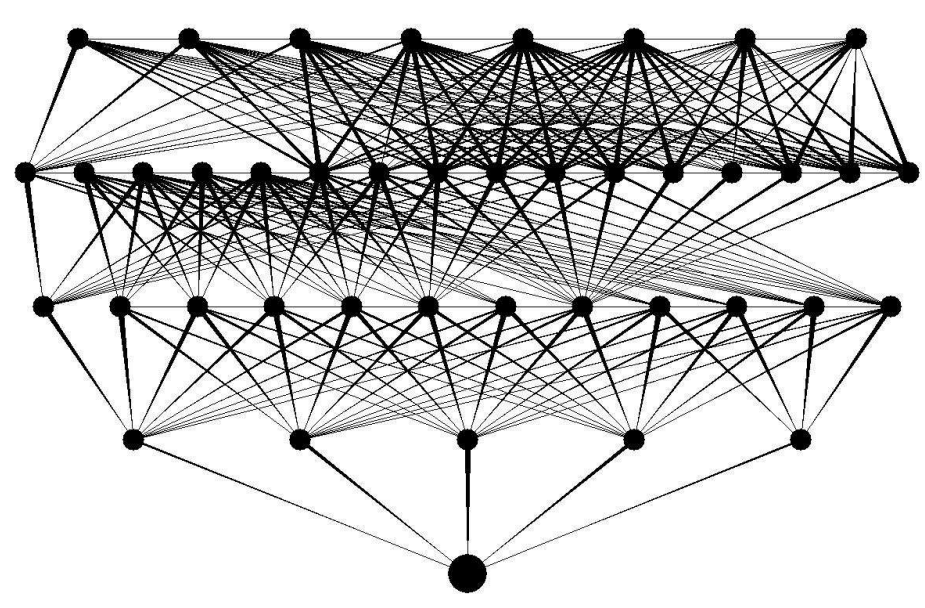

Figure 9: Example of a network created with $S_{\max }=50, \zeta_{n}=0.005, \zeta_{r}=0.006$ and $\zeta_{c}=0$. The thick end of the links points to the predator, indicating that some species on the third trophic level also feed on prey on the fourth level. The single node at the bottom represents the external resources.

Since the asymmetric variation of the parameters in the speciation process decreases the fraction of first level species, the food webs become more prone to larger extinction avalanches again. The size distributions of avalanches for different maximal network sizes are now cut off at larger avalanche sizes that are close the respective maximal network size (figure 10). The distributions now all decay monotonically. The exponents again vary between the curves $\left(S_{\max }=50\right.$ : $\alpha=2.2 \pm 0.1, S_{\max }=100: \alpha=2.5 \pm 0.2$, and $S_{\max }=200: \alpha=2.2 \pm 0.1$ ), but they are now consistently in the range observed in other models [42, 2, 29] and only for $S_{\max }=100$ it appears to be significantly higher than the empirical estimate $[28,33]$.

\begin{tabular}{r|rllrlllll}
$S_{\max }$ & $\bar{S}$ & $\mathrm{TL}$ & $C$ & $L / S$ & $\mathrm{~T}$ & $\mathrm{I}$ & $\mathrm{B}$ & $\mathrm{Can}$ & $\mathrm{Om}$ \\
\hline 50 & 42.4 & 2.13 & 0.20 & 8.4 & 0.18 & 0.68 & 0.15 & 0.21 & 0.85 \\
100 & 85.7 & 2.18 & 0.16 & 13.4 & 0.12 & 0.76 & 0.12 & 0.17 & 0.87 \\
200 & 172.0 & 2.18 & 0.14 & 23.2 & 0.06 & 0.82 & 0.13 & 0.16 & 0.87
\end{tabular}

Table 1: Statistical properties of the food webs generated with asymmetric speciation rules. T, I, and $\mathrm{B}$ are the fractions of top, intermediate, and basal species, respectively, Can is the fraction of cannibalistic species, and $\mathrm{Om}$ is the fraction of omnivorous species. 


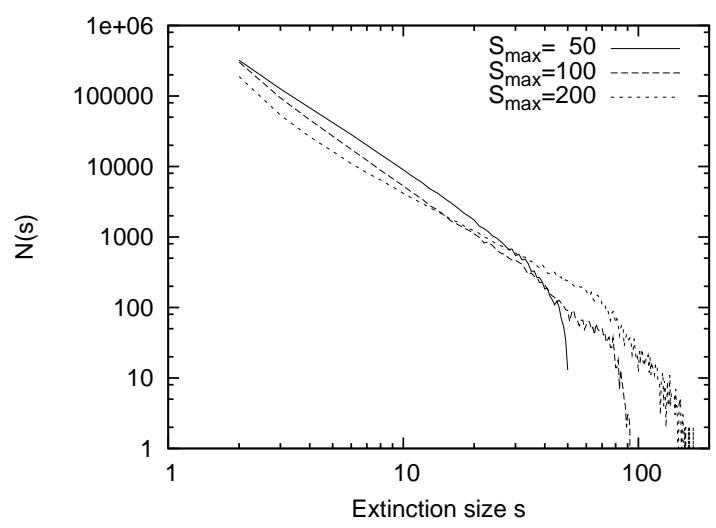

Figure 10: Size distribution of extinction avalanches in networks with $S_{\max }=50,100$, and 200 species when competition acts between species on the same trophic level and speciation is biased towards lower parameter values. Data have been accumulated from time series with $10^{8}$ time steps. The following asymmetry parameters have been used: $S_{\max }=50: \zeta_{n}=0.005, \zeta_{r}=0.006, \zeta_{c}=0$; $S_{\max }=100: \zeta_{n}=0.005, \zeta_{r}=0.007, \zeta_{c}=0 ; S_{\max }=200: \zeta_{n}=0.002, \zeta_{r}=0.008, \zeta_{c}=0.001$.

\section{Discussion}

In this paper a model for food web evolution has been developed that is based on the framework of the niche model. It has proven to be capable of building large, complex food webs that exhibit a number of properties found in empirical food webs. To obtain this result, the basic algorithm had to be refined twice. It was found that the level of stress a species experiences and that determines its extinction probability has to depend on the number of species with the same ecological function. Otherwise the probability that all basal species become extinct by chance is too high to allow for a stable development of the networks. Beyond that, to obtain food webs with food chains longer than two links it was necessary to bias the speciation process so that the parameters of the emerging species are on average smaller than those of its parent species.

The direction of the drift terms can at least in parts be motivated ecologically. For example, one can argue that there must be some pay-off for specialized predators and thus justify the bias towards smaller feeding ranges. The bias towards smaller niche values is more difficult to interpret as the meaning of the niche dimension is not a priory clear. The body size of species has frequently been used to explain the feeding hierarchy in empirical food webs $[47,10]$. By relating the niche value of a species in the model to the average body size of its individuals, the bias towards smaller niche values can be interpreted as a pay-off for not being too much larger than the potential prey. This is supported by empirical data on predator-prey body-size ratios: in nature, predators tend to be only one or two orders of magnitudes larger than their prey [4].

But identifying the niche axis with the body size of the species also is problematic in some respect: The bias of the niche values towards lower values is in contradiction with the observed tendency towards larger body sizes (Cope's rule, $[1,21]$ ). This problem could be overcome by reversing the bias in the body mass and at the same time increasing the risk of extinction with body 
size (niche value) to prevent species from becoming larger and larger. With these assumptions, empirical body size distributions could be reproduced successfully [9]. In the present work, the evolutionary algorithm was not trained to produce realistic body mass distributions, but the emerging distribution of niche values is able to produce food webs with a realistic topology. In this sense, the algorithm employed here represents an effective description of the underlying elementary evolutionary processes.

The exponent of the size distribution of extinction avalanches in this model agrees well with paleontological data and with other models of large-scale evolution for all network sizes that have been analyzed. But it is interesting to note that this only holds for the networks with a realistic topology. When the community is dominated by short food chains, the upper end of the avalanche distributions displays characteristic deviations from the empirical data. This indicates that there is a connection between the topological complexity of a food web and its evolutionary extinction record that has not been seen in previous models.

The degree of asymmetry in the speciation process had to be determined by hand for every maximal food web size $S_{\max }$ that was investigated. However, it should be noted that the values of the parameters $\zeta_{n, r, c}$ have been determined by adjusting the distributions of the species' parameters $n, r$, and $c$ after several ten thousand time steps to their distributions at initialization and not by adjusting the extinction statistics. Both the topology of the food webs and the power law distribution of the avalanche sizes emerge once the parameters of the species on average roughly follow their initial distributions.

Stochastic models of food web evolution are sometimes criticized for being too simplistic. For example, instead of removing whole species by a stochastic process, it is more realistic to compute the population dynamics explicitly. This has been done using stochastic, individual based algorithms $[8,34,35,36]$ or using deterministic equations that determine which species becomes extinct and which species persists in the network. Examples for the latter case are the Webworld model $[13,14]$ or two models that also make use of a 1-dimensional niche axis and contiguous feeding ranges $[22,19]$. In the cited studies, several aspects of the evolutionary history that have been presented here, like intermittent dynamics or the extinction record, have been discussed, too. However, the high model complexity and the need to calculate dynamics on separate time scales make the evaluation of the models difficult. Evolutionary time series simulated by these models typically are much shorter than the ones obtained from stochastic models and data of extinction statistics are less extensive. The results concerning the size distribution of extinction avalanches obtained in different studies are also not conclusive: while it was found to decay exponentially in [13], the data obtained in [19] are consistent with a power law with exponent 2.

When in addition to population dynamics behavioral effects such as adaptive foraging or predator avoidance are considered, the topology of food webs can also change on a much faster time scale than the evolutionary scale. With this approach important insights on the structure [17, 46] or the dynamical behavior and stability $[25,26]$ of food webs can be gained, however, it has rarely been combined with modeling of the evolutionary assembly of the networks (but see [19]). 


\section{Acknowledgements}

Comments and help by Barbara Drossel are gratefully acknowledged. C.G. is supported by the German Research Foundation (BR 2315/9-1).

\section{References}

[1] J. Alroy. Cope's rule and the dynamics of body mass evolution in north american fossil mammals. Science, 280 (1998), 731-734.

[2] L.A. Nunes Amaral, M. Meyer. Environmental changes, coextinction, and patterns in the fossil record. Phys. Rev. Lett., 82 (1999), 652-655.

[3] P. Bak, K. Sneppen. Punctuated equilibrium and criticality in a simple model of evolution. Phys. Rev. Lett., 71 (1993), 4083-4086.

[4] U. Brose, et al. Consumer-resource body-size relationships in natural food webs. Ecology, 87 (2006), 24112417.

[5] J. Camacho, R. Guimerà, L.A. Nunes Amaral. Analytical solution of a model for complex food webs. Phys. Rev. E, 65 (2002), 030901(R).

[6] J. Camacho, R. Guimerà, L.A. Nunes Amaral. Robust patterns in food web structure. Phys. Rev. Lett., 88 (2002), 228102.

[7] M.-F. Cattin, L.-F. Bersier, C. Banašek-Richter, R. Baltensperger, J.-P. Gabriel. Phylogenetic constraints and adaptation explain food-web structure. Nature, 427 (2004), 835-839.

[8] K. Christensen, S.A. Di Collobiano, M. Hall, H.J. Jenssen. Tangled Nature: A model of evolutionary ecology. J. Theor. Biol., 216 (2002), 73-84.

[9] A. Clauset, D.E. Erwin. The evolution and distribution of species body size. Science, 321 (2008), 399-401.

[10] J.E. Cohen, S.L. Pimm, P. Yodzis, J. Saldaña. Body sizes of animal predators and animal prey in food webs. J. Anim. Ecol., 62 (1993), 67-78.

[11] B. Drossel. Extinction events and species lifetimes in a simple ecological model. Phys. Rev. Lett., 81 (1998), 5011-5014.

[12] B. Drossel. Biological evolution and statistical physics. Adv. Phys., 50 (2001), 209-295.

[13] B. Drossel, P.G. Higgs, A.J. McKane. The influence of predator-prey dynamics on the longterm evolution of food web structure. J. Theor. Biol., 208 (2001), 91-107. 
[14] B. Drossel, A.J. McKane, C. Quince. The impact of nonlinear functional responses on the long-term evolution of food web structure. J. Theor. Biol., 229 (2004), 539-548.

[15] J.A. Dunne, R.J. Williams, N.D. Martinez. Network structure and robustness of marine food webs. Mar. Ecol. Prog. Ser., 273 (2004), 291-302.

[16] N. Eldredge, S.J. Gould. In: Models in Paleobiology, Schopf, T.J.M. (Ed.), Freeman, San Francisco, 1972.

[17] J.L. Garcia-Domingo, J. Saldaña. Food-web complexity emerging from ecological dynamics on adaptive networks. J. Theor. Biol., 247 (2007), 819-826.

[18] S.J. Gould, N. Eldredge. Punctuated equilibrium comes of age. Nature, 366 (1993), 223-227.

[19] C. Guill, B. Drossel. Emergence of complexity in evolving niche model food webs. J. Theor. Biol., 251 (2008), 108-120.

[20] G. Hardin. The competitive exclusion principle. Science, 131 (1960), 1292-1297.

[21] D.W.E. Hone, M.J. Benton. The evolution of large size: how does Cope's rule work? Tr. Ecol. Evol., 20 (2005), 4-6.

[22] N. Loeuille, M. Loreau. Evolutionary emergence of size-structured food webs. Proc. Nat. Acad. Sci., 102 (2005), 5761-5766.

[23] B. Kartascheff, C. Guill, B. Drossel. Positive complexity-stability relations in food web models without foraging adaptation. J. Theor. Biol., 259 (2009), 12-23.

[24] S.A. Kauffman, S. Johnsen. Coevolution to the edge of chaos: Coupled fitness landscapes, poised states, and coevolutionary avalanches. J. Theor. Biol., 149 (1991), 467-505.

[25] M. Kondoh. Foraging adaptation and the relationship between food-web complexity and stability. Science, 299 (2003), 1388-1391.

[26] M. Kondoh. Does foraging adaptation create the positive complexity-stability relationship in realistic food-web structure? J. Theor. Biol., 238 (2006), 646-651.

[27] R.M. May. Unanswered questions in ecology. Phil. Trans. R. Soc. Lond. B, 354 (1999), 19511959.

[28] M.E.J. Newman. Self-organized criticality, evolution and the fossil extinction record. Proc. R. Soc. Lond. B, 263 (1996), 1605-1610.

[29] M.E.J. Newman. A model of mass extinction. J. Theor. Biol., 189 (1997), 235-252.

[30] M.E.J. Newman, R.G. Palmer. Models of Extinction: A Review. arXiv:adap-org/ 9908002v1 (1999). 
[31] M. Paczuski, S. Maslov, P. Bak. Avalanche dynamics in evolution, growth, and depinning models. Phys. Rev. E, 53 (1996), 414-443.

[32] D.M. Raup. Biological extinction in earth history. Science, 231 (1986), 1528-1533.

[33] D.M. Raup. A kill curve for phanerozoic marine species. Paleobiology, 17 (1991), 37-48.

[34] P.A. Rikvold. Self-optimization, community stability, and fluctuations in two individual-based models of biological coevolution. J. Math. Biol., 55 (2007),653-677.

[35] P.A. Rikvold, V. Sevim. Individual-based predator-prey model for biological coevolution: Fluctuations, stability, and community structure. Phys. Rev. E, 75 (2007), 051920.

[36] P.A. Rikvold. Complex dynamics in coevolution models with ratio-dependent functional response. Ecol. Comp. (2009), in press.

[37] A.G. Rossberg, H. Matsuda, T. Amemiya, K. Itoh. An explanatory model for food-web structure and evolution. Ecol. Comp., 2 (2005), 312-321.

[38] A.G. Rossberg, H. Matsuda, T. Amemiya, K. Itoh. Food webs: Experts consuming families of experts. J. Theor. Biol., 241 (2006), 552-563.

[39] A.G. Rossberg, R. Ishii, T. Amemiya, K. Itoh. The top-down mechanism for body-massabundance scaling. Ecology, 89 (2008), 567-580.

[40] F. Slanina, M. Kotrla. Extremal dynamics model on evolving networks. Phys. Rev. Lett., 83 (1999), 5587-5590.

[41] R.V. Solé, J. Bascompte. Are critical phenomena relevant to large-scale evolution? Proc. R. Soc. Lond. B, 263 (1996), 161-168.

[42] R.V. Solé, S.C. Manrubia. Extinction and self-organized criticality in a model of large-scale evolution. Phys. Rev. E, 54 (1996), R42-R45.

[43] R.V. Solé, S.C. Manrubia, M. Benton, P. Bak. Self-similarity of extinction statistics in the fossil record. Nature, 388 (1997), 764-767.

[44] D.B. Stouffer, J. Camacho, R. Guimerà, C.A. Ng, L.A. Nunes Amaral. Quantitative patterns in the structure of model and empirical food webs. Ecology, 86 (2005), 1301-1311.

[45] D.B. Stouffer, J. Camacho, L.A. Nunes Amaral. A robust measure of food web intervality. Proc. Nat. Acad. Sci., 103 (2006), 19015-19020.

[46] S. Uchida, B. Drossel, U. Brose. The structure of food webs with adaptive behaviour. Ecol. Mod., 206 (2007), 263-276. 
[47] P.H. Warren, J.H. Lawton. Invertebrate predator-prey body size relationships: an explanation for upper triangular food webs and patterns in food web structure? Oecologia, 74 (1987), 231-235.

[48] E.P. White, B.J. Enquist, J.L. Green. On estimating the exponent of power-law frequency distributions. Ecology, 89 (2008), 905-912.

[49] R.J. Williams, N.D. Martinez. Simple rules yield complex food webs. Nature, 404 (2000), $180-183$

[50] R.J. Williams, N.D. Martinez. Success and its limits among structural models of complex food webs. J. Anim. Ecol., 77 (2008), 512-519. 